\title{
Spatio-temporal Assimilation of Modelled \\ Catchment Loads with Monitoring Data in the Great Barrier Reef
}

Daniel W. Gladish ${ }^{1}$, Petra M. Kuhnert ${ }^{1}$, Daniel E. Pagendam ${ }^{1}$, Christopher K. Wikle ${ }^{2}$, Rebecca Bartley ${ }^{1}$, Ross D. Searle ${ }^{1}$, Robin J. Ellis ${ }^{3}$, Cameron Dougall $^{4}$, Ryan D.R. Turner ${ }^{3}$, Stephen E. Lewis ${ }^{5}$, Zoë T. Bainbridge ${ }^{5}$, and Jon E. Brodie ${ }^{5}$

${ }^{1}$ Commonwealth Scientific and Industrial Research Organisation (CSIRO) ${ }^{2}$ University of Missouri

${ }^{3}$ Department of Science, Information Technology, and Innovation (DSITI)

${ }^{4}$ Department of Natural Resources and Mines

${ }^{5}$ James Cook University

\section{Web Supplement: Monitoring Data and Covariates}

We present in this section an overview of the available monitoring data and the covariates of interest for the flow and concentration model runs. Figure 1 shows the spatial distribution of the $\mathrm{C}$-factor (and hence vegetative cover), the covariate of interest for the 
concentration model for 1990/1991 (top left) and 2006/2007 (top right) financial years (FY). These specific values for the $\mathrm{C}$-factor are based on variable estimates of cover called the Bare Ground Index (Byrne et al., 2004). Of note, while there are changes in C-factor from year to year, they are minimal. The covariate incorporated in the model for flow consisted of the annual mean Pacific Decadal Oscillation (PDO). We summarise PDO in the bottom of Figure 1 for all years modelled. Note that PDO is a monthly variable (Mantua et al. 1997). For our purposes, we aggregate PDO to the financial year level by averaging from July to June for each FY.

Available monitoring data is sparse for the Upper Burdekin, with flow and concentration data available only at 8 of the available 411 sites, with an additional site containing only flow data and 5 additional sites containing concentration data. Furthermore, concentration is significantly more sparse in available monitoring than flow. Figure 2 shows the available concentration data while Figure 3 shows the available flow monitoring data for all available monitored sites. The corresponding D-SedNet output is also included for reference.

\section{Web Supplement: Posterior Distribution Results}

In this section we present the results of the posterior distribution for the parameters associated with the dynamics of latent process $\mathbf{Y}_{i k}$. Influencing the dynamics of the $\mathbf{Y}_{i k}$ process are the underlying empirical orthogonal function (EOF) decomposition parameters M, $\Sigma_{\eta}$, and $\sigma_{\gamma}^{2}(l)$. Tables 1 and 2 show the posterior means and $95 \%$ credible intervals for the concentration and flow model parameters. Particularly noteworthy are the $2^{\text {nd }}, 7^{\text {th }}, 8^{\text {th }}$,

and $11^{\text {th }}$ values of $\mathbf{M}^{C}$ for concentration that are significantly different from both 0 and 1. Significant values of the $\mathrm{M}^{Q}$ matrix for flow are also noted for the $1^{\text {st }}, 3^{\text {rd }}, 4^{\text {th }}$, and $5^{\text {th }}$ values. These indicate that the respective components of $\boldsymbol{\beta}_{k}$ do not follow a white noise nor a random walk process and tend to have a stationary autoregressive structure. This gives evidence for modelling the evolution of the spatial process dynamically, and thus a reason 
for modelling the dynamics of $\mathbf{Y}_{i k}$ in a reduced rank setting.

Table 3 show the posterior results for the covariate coefficients corresponding to the C-factor and PDO, the covariates of interest as noted in Section 3.7 of the manuscript. These values influence the latent process $\mathbf{Y}_{i k}$ through the seasonal EOFs $\Psi$. As such, they influence each day of the year differently. As noticed in the main manuscript, a physical interpretation of the covariate coefficients is best achieved through averaging to the yearly scale. All individual components of the covariate coefficient for concentration are significantly different from zero while all but the $5^{\text {th }}$ component are significantly different for flow.

\section{Web Supplement: Markov Chain Monte Carlo Algo- rithm}

We use a standard Markov Chain Monte Carlo (MCMC) algorithm with Gibbs updates to obtain samples from the posterior distribution of our model. We let $[Y \mid X]$ and $[X]$ denote the conditional probability distribution and unconditional probability distribution of $Y$ given $X$ and of $X$, respectively. Recall that $q_{\epsilon}(j), r_{\epsilon}(j),(j=1, \ldots, T), q_{\gamma}(l)$, $r_{\gamma}(l)(l=1, \ldots, p), \boldsymbol{\lambda}_{0}, \sigma_{\lambda}^{2}, \boldsymbol{\mu}_{M}, \boldsymbol{\Sigma}_{M}, q_{b}(l), r_{b}(l)(l=1, \ldots, q), \boldsymbol{\mu}_{0}$, and $\boldsymbol{\Sigma}_{0}$ are pre-defined hyperparameters.

First, for $i=1, \ldots, n$ and $k=1, \ldots, K$, the full conditional for $\mathbf{Y}_{i k}$ is given by

$$
\left[\mathbf{Y}_{i k} \mid \cdot\right] \sim \operatorname{Gau}\left(\mathbf{V}_{i k}^{-1} \mathbf{a}_{i k}, \mathbf{V}_{i k}^{-1}\right)
$$

where

$$
\mathbf{V}_{i k}=\frac{1}{\sigma_{o b s}^{2}} \mathbf{H}_{i k}^{o b s \prime} \mathbf{H}_{i k}^{o b s}+\left(\boldsymbol{\Sigma}_{i k}^{s r c}\right)^{-1}+\boldsymbol{\Sigma}_{\epsilon}^{-1}
$$


and

$$
\mathbf{a}_{i k}=\frac{1}{\sigma_{o b s}^{2}} \mathbf{H}_{i k}^{o b s \prime} \mathbf{Z}_{i k}^{o b s}+\left(\boldsymbol{\Sigma}_{i k}^{s r c}\right)^{-1} \mathbf{Z}_{i k}^{s r c}+\boldsymbol{\Sigma}_{\epsilon}^{-1} \boldsymbol{\Psi} \boldsymbol{\alpha}_{i k} .
$$

The full conditional for $\sigma_{\epsilon}^{2}(j)$ for $j=1, \ldots, T$ is

$$
\left[\sigma_{\epsilon}^{2}(l) \mid \cdot\right] \sim \operatorname{IG}\left(q_{\epsilon, 0}(j), r_{\epsilon, 0}(j)\right)
$$

where

$$
q_{\epsilon, 0}(j)=\frac{n K}{2}+q_{\epsilon}
$$

and

$$
r_{\epsilon, 0}(j)=\left(\frac{1}{r_{\epsilon}}+\frac{1}{2} \sum_{i=1}^{n} \sum_{k=1}^{K}\left(Y_{i j k}-\boldsymbol{\psi}(j)^{\prime} \boldsymbol{\alpha}_{i k}\right)^{2}\right)^{-1}
$$

with $\psi(j)$ the $j$ th row of the matrix $\Psi$.

The full conditional for $\widetilde{\boldsymbol{\alpha}}_{k}$ for $k=1, \ldots, K$ is

$$
\left[\widetilde{\boldsymbol{\alpha}}_{k} \mid \cdot\right] \sim \operatorname{Gau}\left(\mathbf{V}_{\alpha, k}^{-1} \mathbf{a}_{\alpha, k}, \mathbf{V}_{\alpha, k}^{-1}\right)
$$

where

$$
\mathbf{V}_{\alpha, k}=\boldsymbol{\Sigma}_{\gamma}^{-1}+\Psi^{\prime} \boldsymbol{\Sigma}_{\epsilon}^{-1} \boldsymbol{\Psi} \otimes \mathbf{I}_{n}
$$

and

$$
\mathbf{a}_{\alpha, k}=\boldsymbol{\Sigma}_{\gamma}^{-1}\left(\boldsymbol{\Phi} \boldsymbol{\beta}+\boldsymbol{\lambda} \otimes \mathbf{X}_{k}\right)+\widetilde{\mathbf{Y}}_{k}
$$

with $\widetilde{\mathbf{Y}}_{k}=\left(\widetilde{\mathbf{Y}}_{1 k}^{\prime}, \ldots, \widetilde{\mathbf{Y}}_{n k}^{\prime}\right)^{\prime}$ and $\widetilde{\mathbf{Y}}_{i k}=\Psi^{\prime} \Sigma_{\epsilon}^{-1} \mathbf{Y}_{i k}$

For the variances of the latent multivariate spatio-temporal field $\sigma_{\gamma}^{2}(l)$ for $l=1, \ldots, p$, the full conditionals

$$
\left[\sigma_{\gamma}^{2}(l) \mid \cdot\right] \sim \operatorname{IG}\left(q_{\gamma, 0}(j), r_{\gamma, 0}(j)\right)
$$


where

$$
q_{\gamma, 0}(j)=q_{\gamma}(j)+\frac{n K}{2}
$$

and

$$
r_{\gamma, 0}(j)=\left\{\frac{1}{r_{\gamma}(j)}+\frac{1}{2} \sum_{k=1}^{K}\left(\widetilde{\alpha}_{k}(l)-\boldsymbol{\Phi}^{(l)} \boldsymbol{\beta}_{k}-\lambda(l) \mathbf{X}_{k}\right)^{\prime}\left(\widetilde{\alpha}_{k}(l)-\boldsymbol{\Phi}^{(l)} \boldsymbol{\beta}_{k}-\lambda(l) \mathbf{X}_{k}\right)\right\}
$$

with $\widetilde{\alpha}_{k}(l)$ correspond to $n$ dimensional vector of the $l$ th spatial field in $\widetilde{\alpha}_{k}, \lambda(l)$ the $l$ th component of $\boldsymbol{\lambda}$, and $\boldsymbol{\Phi}^{(l)}$ the corresponding $n \times q$ matrix, where

$$
\boldsymbol{\Phi} \equiv\left(\begin{array}{c}
\boldsymbol{\Phi}^{(1)} \\
\vdots \\
\boldsymbol{\Phi}^{(p)}
\end{array}\right)
$$

The full conditional for the covariate coefficient vector $\lambda$ can be defined through the following expression:

$$
\begin{aligned}
\widetilde{X} & =\sum_{k=1}^{K} \mathbf{X}_{k}^{\prime} \mathbf{X}_{\mathbf{k}}, \\
\widetilde{\mathbf{X}}_{k} & =\left(\mathbf{X}_{k}, \ldots, \mathbf{X}_{k}\right)_{n p}^{\prime}, \\
\widetilde{\boldsymbol{\Sigma}}_{\gamma} & =\operatorname{diag}\left(\sigma_{\gamma}^{2}(1), \ldots, \sigma_{\gamma}^{2}(p)\right)
\end{aligned}
$$

Further, define the following $n p$ dimensional vector $\widetilde{\lambda}$ as

$$
\widetilde{\boldsymbol{\lambda}}=\boldsymbol{\Sigma}_{\gamma}^{-1} \sum_{k=1}^{K}\left(\widetilde{\boldsymbol{\alpha}}_{k}-\boldsymbol{\Phi} \boldsymbol{\beta}_{k}\right)
$$

Then, the full conditional of $\boldsymbol{\lambda}$ has the form

$$
[\boldsymbol{\lambda} \mid \cdot] \sim \operatorname{Gau}\left(\mathbf{V}_{\lambda}^{-1} \mathbf{a}_{\lambda}, \mathbf{V}_{\lambda}^{-1}\right)
$$


where

$$
\mathbf{V}_{\lambda}=\tilde{X} \widetilde{\Sigma}_{\gamma}^{-1}+\frac{1}{\sigma_{\lambda}^{2}} \mathbf{I}
$$

and

$$
\mathbf{a}_{\lambda}=\left(\mathbf{I}_{p} \otimes \mathbf{1}_{n}^{\prime}\right) \tilde{\boldsymbol{\lambda}}
$$

where $\mathbf{I}_{p}$ is the $p \times p$ dimensional identity matrix, and $\mathbf{1}_{n}$ is the $n$-dimensional vector of ones.

The full conditional for the initialisation vector $\boldsymbol{\beta}_{0}$ is straightforward with the form

$$
\left[\boldsymbol{\beta}_{0} \mid \cdot\right] \sim \operatorname{Gau}\left(\mathbf{V}_{0}^{-1} \mathbf{a}_{0}, \mathbf{V}_{0}^{-1}\right)
$$

where

$$
\mathbf{V}_{0}=\mathbf{M}^{\prime} \boldsymbol{\Sigma}_{\eta}^{-1} \mathbf{M}+\boldsymbol{\Sigma}_{0}^{-1}
$$

and

$$
\mathbf{a}_{0}=\mathbf{M}^{\prime} \boldsymbol{\Sigma}_{\eta}^{-1} \boldsymbol{\beta}_{1}+\boldsymbol{\Sigma}_{0}^{-1} \boldsymbol{\mu}_{0}
$$

The full conditional for the expansion coefficients $\boldsymbol{\beta}_{k}$, for $k=1, \ldots, K-1$

$$
\left[\boldsymbol{\beta}_{k} \mid \cdot\right] \sim \operatorname{Gau}\left(\mathbf{V}_{k}^{-1} \mathbf{a}_{k}, \mathbf{V}_{k}^{-1}\right)
$$

where

$$
\mathbf{V}_{k}=\Sigma_{\eta}^{-1}+\mathbf{M}^{\prime} \Sigma_{\eta}^{-1} \mathbf{M}+\boldsymbol{\Phi}^{\prime} \boldsymbol{\Sigma}_{\gamma}^{-1} \boldsymbol{\Phi}
$$

and

$$
\mathbf{a}_{k}=\boldsymbol{\Sigma}_{\eta}^{-1} \mathbf{M} \boldsymbol{\beta}_{k-1}+\boldsymbol{\Sigma}_{\eta}^{-1}+\mathbf{M}^{\prime} \boldsymbol{\Sigma}_{\eta}^{-1} \boldsymbol{\beta}_{k+1}+\boldsymbol{\Phi} \boldsymbol{\Sigma}_{\gamma}^{-1}\left(\widetilde{\boldsymbol{\alpha}}_{k}-\boldsymbol{\lambda} \otimes \mathbf{X}_{k}\right)
$$

The full conditional for the expansion coefficients $\boldsymbol{\beta}_{K}$ is

$$
\left[\boldsymbol{\beta}_{K} \mid \cdot\right] \sim \operatorname{Gau}\left(\mathbf{V}_{K}^{-1} \mathbf{a}_{K}, \mathbf{V}_{K}^{-1}\right)
$$


where

$$
\mathbf{V}_{K}=\Sigma_{\eta}^{-1}+\boldsymbol{\Phi}^{\prime} \boldsymbol{\Sigma}_{\gamma}^{-1} \boldsymbol{\Phi}
$$

and

$$
\mathbf{a}_{K}=\boldsymbol{\Sigma}_{\eta}^{-1} \mathbf{M} \boldsymbol{\beta}_{K-1}+\boldsymbol{\Sigma}_{\eta}^{-1}+\mathbf{\Phi} \boldsymbol{\Sigma}_{\gamma}^{-1}\left(\widetilde{\boldsymbol{\alpha}}_{K}-\boldsymbol{\lambda} \otimes \mathbf{X}_{K}\right)
$$

Define $\mathbf{m} \equiv \operatorname{diag}(\mathbf{M})$. Let $\mathbf{m} \equiv(m(1), \ldots, m(q))^{\prime}$. Then, the full conditional for the propagator parameters $m(l), l=1, \ldots, q$ follows as

$$
[m(l) \mid \cdot] \sim \operatorname{Gau}\left(V_{m}(l)^{-1} a_{m}(l), V_{m}(l)^{-1}\right)
$$

where $V_{m}(l)=\sum_{k=0}^{K-1}\left(\beta_{k}(l)^{2} / \sigma_{\eta}^{2}(l)\right)+1 / \sigma_{M}^{2}(l)$ and $a_{m}(l)=\sum_{k=1}^{K}\left(\beta_{k}(l) \beta_{k-1}(l) / \sigma_{\eta}^{2}(l)\right)+$ $\mu_{m}(l) / \sigma_{M}^{2}(l)$. Note that $\mu_{m}(l)$ is the $l$ th element of $\boldsymbol{\mu}_{M}$, and $\sigma_{M}^{2}(l)$ is the $l$ th diagonal element of the matrix $\Sigma_{M}$.

Lastly, the variances of the autoregression model $\sigma_{\eta}^{2}(l)$, for $l=1, \ldots, q$, have the full conditional distribution

$$
\left[\sigma_{\eta}^{2}(l) \mid \cdot\right] \sim \operatorname{IG}\left(q_{\eta, 0}(l), r_{\eta, 0}(l)\right)
$$

where

$$
q_{\eta, 0}(l)=q_{\eta}(l)+\frac{K}{2}
$$

and

$$
r_{\eta, 0}(l)=\left(\frac{1}{r_{\eta}(l)}+\frac{1}{2} \sum_{k=1}^{K}\left(\beta_{k}(l)-m(l) \beta_{k-1}(l)\right)^{2}\right)^{-1}
$$




\section{References}

Byrne, M., Hassett, R., Taube, C., and Henry, B. (2004). Objective monitoring of ground cover across the desert channels queensland region. Department of Natural Resources and Mines, Brisbane, Australia.

Mantua, N., Hare, S., Zhang, Y., Wallace, J., and Francis, R. (1997). A pacific interdecadal climate oscillation with impacts on salmon production. Bulletin of the American Meteorological Society, 78(6):1069-1079. Data obtained from http: / /www . reefplan . qld.gov.au/resources/assets/reef-plan-2013.pdf. Accessed: 201502-23. 


\section{C-Factor}
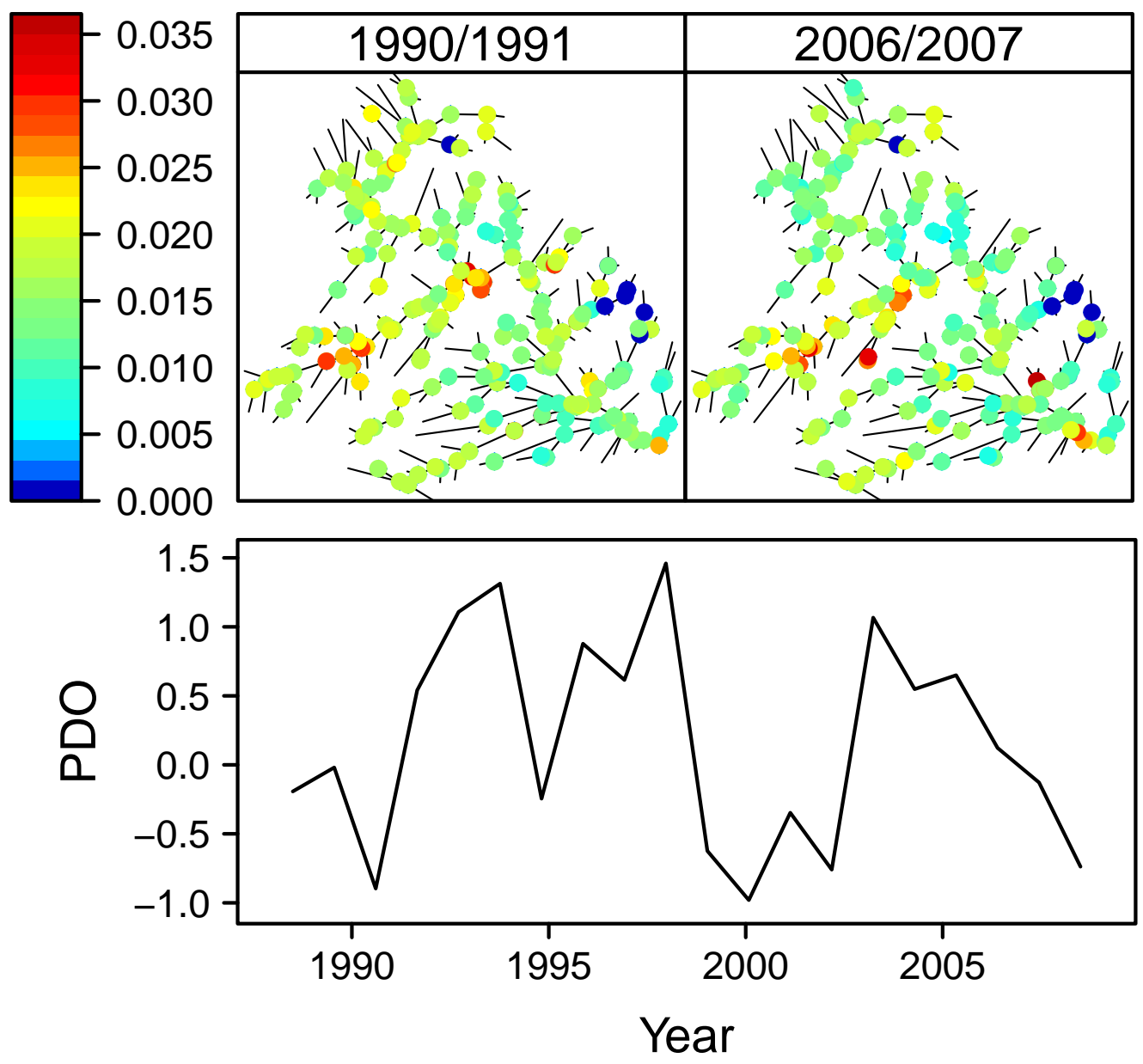

Figure 1: Image of values for average $\mathrm{C}$-factor at the 411 sites in the Upper Burdekin for FY 1990/1991 (upper left) and FY 2006/2007 (upper right), and annual mean PDO by year (bottom). Note that $\mathrm{C}$-factor is used as an analogue for vegetative cover. 

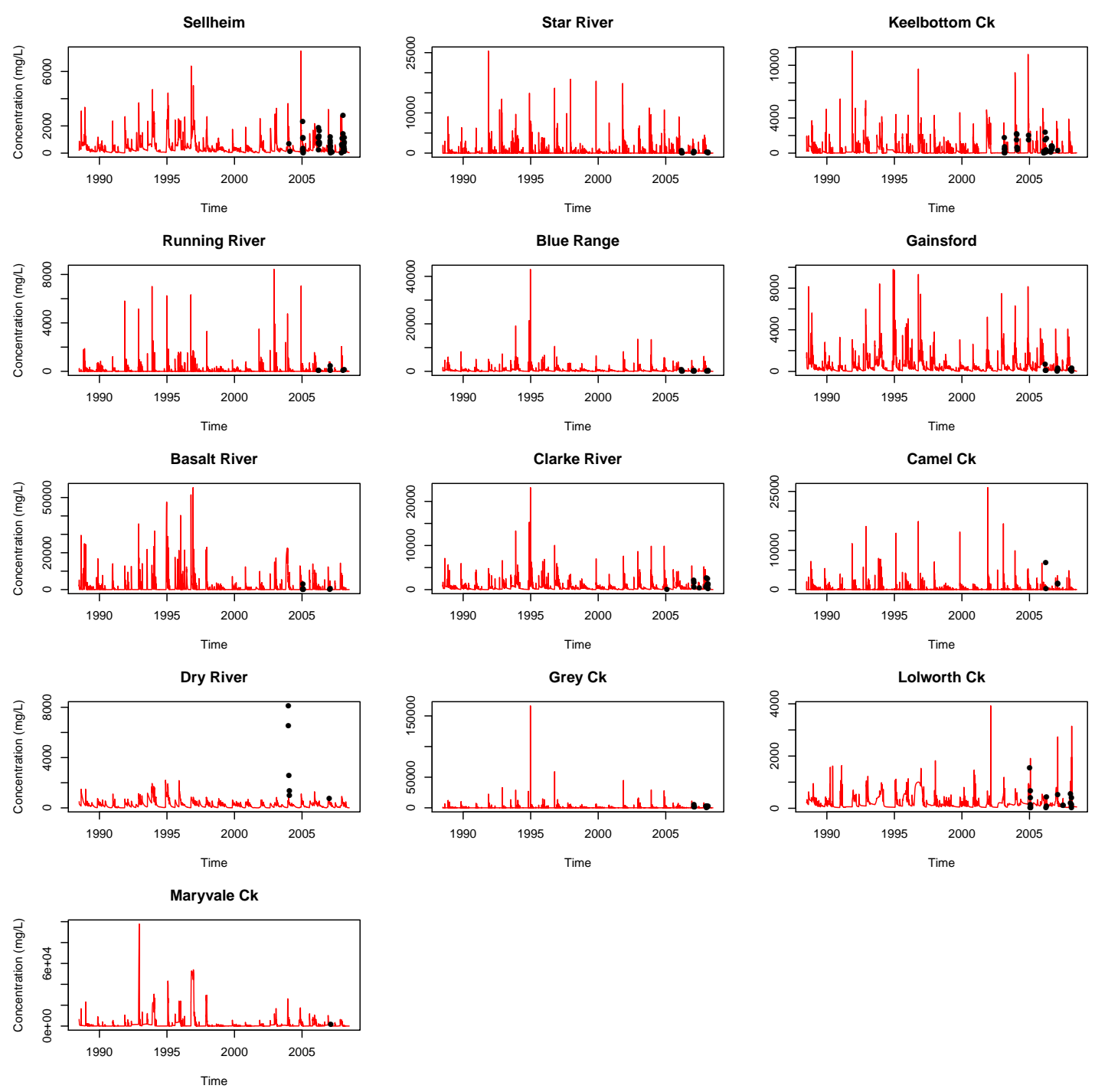

Figure 2: Time series plots of D-SedNet model output (red line) and monitoring data (black points) all sites with monitored concentration in $\mathrm{mg} / \mathrm{L}$. The sites (as marked in Figure 1 in the manuscript) are Sellheim, Star River, Keelbottom Creek, Running River, Blue Range, Gainsford, Basalt River, Clarke River, Camel Creek, Dry River, Grey Creek, Lolworth Creek, and Maryvale Creek. The first 8 sites also have monitoring data for flow while the remaining 5 do not. 

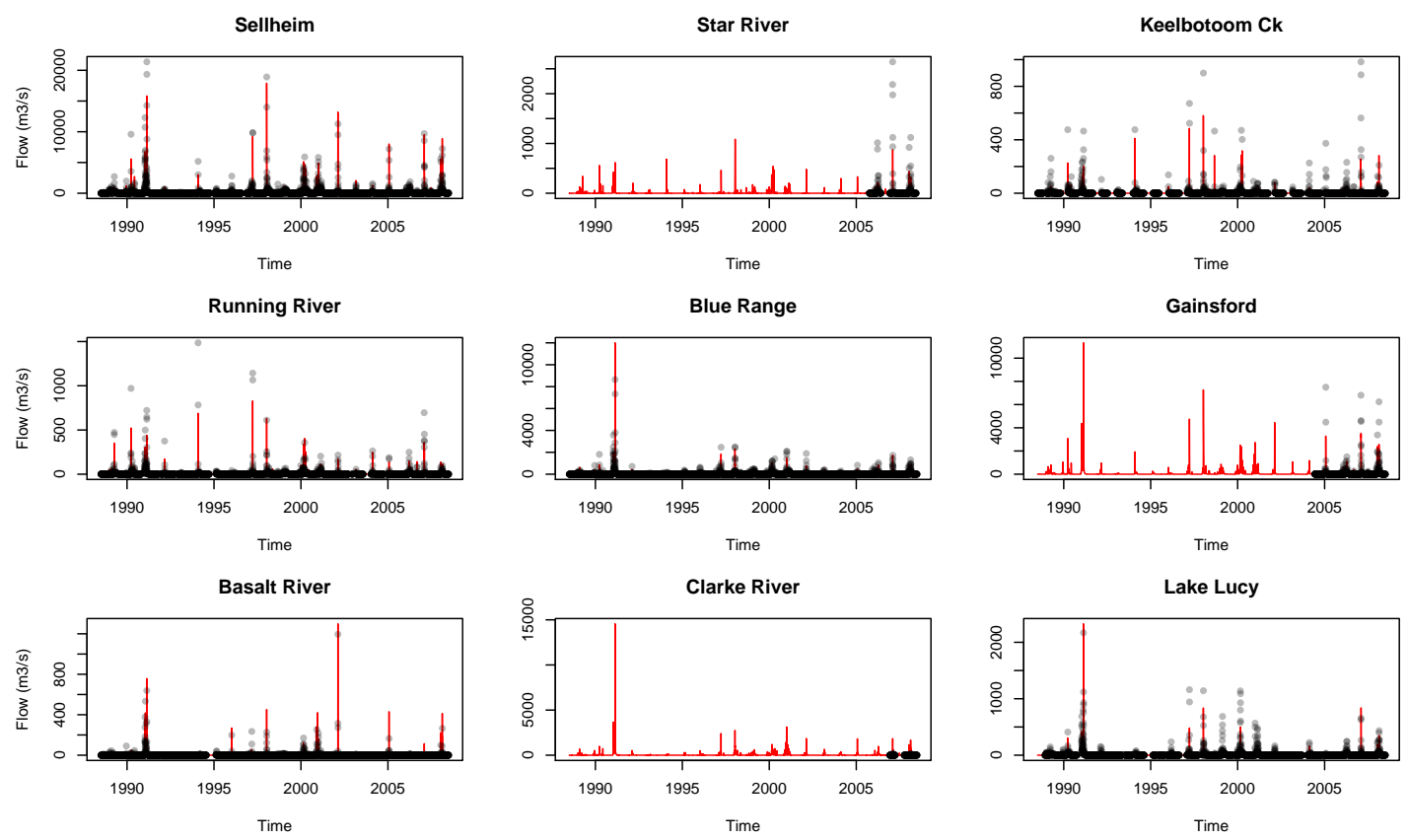

Figure 3: Time series plots of D-SedNet model output (red line) and monitoring data (black points) all sites with monitored flow in $\mathrm{m}^{3} / \mathrm{s}$. The sites (as marked in Figure 1 in the manuscript) are Sellheim, Star River, Keelbottom Creek, Running River, Blue Range, Gainsford, Basalt River, Clarke River, Camel Creek, and Lake Lucy. All but Lake Lucy also have monitoring data for concentration. 


\begin{tabular}{|c|c|c|c|}
\hline Parameter & Mean & Lower bound & Upper Bound \\
\hline$M_{1}^{C}$ & -0.06 & -0.40 & 0.25 \\
\hline$M_{2}^{C}$ & 0.38 & 0.08 & 0.65 \\
\hline$M_{3}^{C}$ & 0.08 & -0.25 & 0.40 \\
\hline$\stackrel{\dot{M}}{C}$ & -0.02 & -0.34 & 0.30 \\
\hline$M_{5}^{C}$ & 0.01 & -0.33 & 0.32 \\
\hline$M_{6}^{C}$ & -0.24 & -0.53 & 0.06 \\
\hline$M_{7}^{C}$ & 0.38 & 0.10 & 0.69 \\
\hline$M_{8}^{C}$ & 0.48 & 0.15 & 0.77 \\
\hline$\stackrel{\stackrel{C}{C}}{C}$ & 0.19 & -0.13 & 0.49 \\
\hline$M_{10}^{C}$ & 0.04 & -0.33 & 0.37 \\
\hline$M_{11}^{C}$ & -0.30 & -0.58 & -0.01 \\
\hline $\boldsymbol{\Sigma}_{\eta}(1)^{C}$ & 191457.30 & 124882.97 & 301707.36 \\
\hline $\boldsymbol{\Sigma}_{\eta}(2)^{C}$ & 127103.15 & 81046.23 & 203864.38 \\
\hline $\boldsymbol{\Sigma}_{\eta}(3)^{C}$ & 77260.69 & 48950.21 & 117290.17 \\
\hline $\boldsymbol{\Sigma}_{\eta}(4)^{C}$ & 67431.38 & 42941.71 & 103418.34 \\
\hline $\boldsymbol{\Sigma}_{\eta}(5)^{C}$ & 74340.70 & 46032.11 & 116967.18 \\
\hline $\boldsymbol{\Sigma}_{\eta}(6)^{C}$ & 52314.65 & 33800.92 & 79339.90 \\
\hline $\boldsymbol{\Sigma}_{\eta}(7)^{C}$ & 43821.04 & 28644.94 & 66549.19 \\
\hline $\boldsymbol{\Sigma}_{\eta}(8)^{C}$ & 52847.50 & 34077.69 & 81377.25 \\
\hline $\boldsymbol{\Sigma}_{\eta}(9)^{C}$ & 39940.39 & 26225.18 & 62573.63 \\
\hline $\boldsymbol{\Sigma}_{\eta}(10)^{C}$ & 49939.05 & 31720.56 & 77919.47 \\
\hline $\boldsymbol{\Sigma}_{\eta}(11)^{C}$ & 28005.92 & 18392.61 & 44841.97 \\
\hline$\sigma_{\gamma, C}^{2}(1)$ & 43553.00 & 42181.00 & 44937.00 \\
\hline$\sigma_{\gamma C}^{2}(2)$ & 1502.20 & 1446.10 & 1559.70 \\
\hline$\sigma_{\gamma, C}^{2}(3)$ & 416.20 & 390.63 & 441.64 \\
\hline$\sigma_{\gamma, C}^{2}(4)$ & 760.66 & 723.16 & 796.83 \\
\hline$\sigma_{\gamma C}^{2}(5)$ & 93.77 & 90.02 & 97.95 \\
\hline$\sigma_{\gamma, C}^{2}(6)$ & 120.26 & 115.31 & 126.26 \\
\hline$\sigma_{\gamma, C}^{2}(7)$ & 31.87 & 30.15 & 33.87 \\
\hline$\sigma_{\gamma C}^{2}(8)$ & 29.72 & 27.77 & 31.59 \\
\hline$\sigma_{\gamma, C}^{2}(9)$ & 31.65 & 29.68 & 33.63 \\
\hline$\sigma_{\gamma, C}^{2}(10)$ & 23.58 & 22.16 & 25.07 \\
\hline$\sigma_{\gamma C}^{2}(11)$ & 50.26 & 47.39 & 53.49 \\
\hline$\sigma_{\gamma, C}^{2}(12)$ & 56.07 & 53.32 & 59.04 \\
\hline$\sigma_{\gamma C}^{2}(13)$ & 22.89 & 21.11 & 24.64 \\
\hline
\end{tabular}

Table 1: The posterior mean and 95\% credible intervals (lower bound, upper bound) for $\mathbf{M}^{C}, \Sigma_{\eta}^{C}$, and $\sigma_{\gamma, C}^{2}$ for the concentration model. 


\begin{tabular}{r|rrr}
\hline Parameter & Mean & Lower bound & Upper Bound \\
\hline$M_{1}^{Q}$ & 0.76 & 0.54 & 0.98 \\
$M_{2}^{Q}$ & 0.26 & -0.11 & 0.61 \\
$M_{3}^{Q}$ & 0.46 & 0.17 & 0.77 \\
$M_{4}^{Q}$ & 0.71 & 0.48 & 0.95 \\
$M_{5}^{Q}$ & 0.41 & 0.09 & 0.73 \\
$M_{6}^{Q}$ & 0.19 & -0.14 & 0.51 \\
$\Sigma_{\eta}(1)^{Q}$ & 236124.28 & 150630.89 & 382358.55 \\
$\Sigma_{\eta}(2)^{Q}$ & 149001.19 & 94215.33 & 229674.38 \\
$\Sigma_{\eta}(3)^{Q}$ & 129009.35 & 79580.70 & 205465.96 \\
$\Sigma_{\eta}(4)^{Q}$ & 66434.58 & 41709.53 & 105473.24 \\
$\Sigma_{\eta}(5)^{Q}$ & 40741.24 & 25926.15 & 62569.62 \\
$\Sigma_{\eta}(6)^{Q}$ & 23560.10 & 14986.56 & 36193.39 \\
$\sigma_{\gamma, Q}^{2}(1)$ & 1550.00 & 1480.00 & 1627.00 \\
$\sigma_{\gamma, Q}^{2}(2)$ & 55.32 & 53.05 & 57.62 \\
$\sigma_{\gamma, Q}^{2}(3)$ & 72.70 & 70.16 & 75.52 \\
$\sigma_{\gamma, Q}^{2}(4)$ & 66.27 & 63.66 & 69.12 \\
$\sigma_{\gamma, Q}^{2}(5)$ & 22.99 & 21.96 & 24.11 \\
$\sigma_{\gamma, Q}^{2}(6)$ & 106.10 & 98.56 & 114.30 \\
$\sigma_{\gamma, Q}^{2}(7)$ & 9.06 & 8.54 & 9.60 \\
$\sigma_{\gamma, Q}^{2}(8)$ & 82.01 & 79.12 & 84.94 \\
\hline
\end{tabular}

Table 2: The posterior mean and $95 \%$ credible intervals (lower bound, upper bound) for $\mathbf{M}^{Q}, \Sigma_{\eta}^{Q}$, and $\sigma_{\gamma, Q}^{2}$ for the flow model. 


\begin{tabular}{r|rrrr}
\hline Parameter & Mean & Lower bound & Upper Bound & \\
\hline$\lambda_{1}^{C}$ & -91.05 & -109.73 & -70.82 & $*$ \\
$\lambda_{2}^{C}$ & -322.74 & -339.47 & -304.79 & $*$ \\
$\lambda_{3}^{C}$ & -486.66 & -502.36 & -472.43 & $*$ \\
$\lambda_{4}^{C}$ & -428.44 & -444.57 & -412.13 & $*$ \\
$\lambda_{5}^{C}$ & 174.67 & 165.31 & 184.16 & $*$ \\
$\lambda_{6}^{C}$ & 297.37 & 286.54 & 308.20 & $*$ \\
$\lambda_{7}^{C}$ & -63.17 & -70.53 & -55.91 & $*$ \\
$\lambda_{8}^{C}$ & -67.03 & -75.17 & -59.07 & $*$ \\
$\lambda_{9}^{C}$ & 49.91 & 42.08 & 57.44 & $*$ \\
$\lambda_{10}^{C}$ & 56.65 & 49.55 & 63.62 & $*$ \\
$\lambda_{11}^{C}$ & 156.97 & 149.54 & 164.33 & $*$ \\
$\lambda_{12}^{C}$ & 110.08 & 102.89 & 117.13 & $*$ \\
$\lambda_{13}^{C}$ & -37.78 & -43.87 & -31.62 & $*$ \\
$\lambda_{1}^{Q}$ & -9.05 & -11.00 & -7.13 & $*$ \\
$\lambda_{2}^{Q}$ & 6.19 & 5.22 & 7.08 & $*$ \\
$\lambda_{3}^{Q}$ & -4.64 & -5.40 & -3.90 & $*$ \\
$\lambda_{4}^{Q}$ & -6.34 & -7.06 & -5.56 & $*$ \\
$\lambda_{5}^{Q}$ & -0.45 & -1.05 & 0.20 & \\
$\lambda_{6}^{Q}$ & 1.96 & 1.54 & 2.42 & $*$ \\
$\lambda_{7}^{Q}$ & 0.41 & 0.00 & 0.86 & $*$ \\
$\lambda_{8}^{Q}$ & -1.38 & -1.68 & -1.09 & $*$ \\
\hline & & & &
\end{tabular}

Table 3: The posterior mean and 95\% credible intervals (lower bound, upper bound) for the covariate coefficient $\boldsymbol{\lambda}^{C}$ for the concentration model (corresponding to cover) and $\boldsymbol{\lambda}^{Q}$ for the flow model (corresponding to PDO). Significant results are indicated by a *. 